\title{
CIRCULAR SUMMABILITY $C$ OF DOUBLE TRIGONOMETRIC SERIES $\left({ }^{1}\right)$
}

BY

\author{
VICTOR L. SHAPIRO
}

1. Introduction. Let $T=\sum a_{m n} e^{i(m x+n y)}$ be a double trigonometric series where $a_{m n}$ are arbitrary complex numbers. Form the circular partial sums

$$
S_{R}(x, y)=\sum_{\left(m^{2}+n^{2}\right)^{1 / 2} \leqq R} a_{m n} e^{i(m x+n y)} .
$$

The series will be said to be circular convergent at the point $(x, y)$ to the finite value $L(x, y)$, if

$$
\lim _{R \rightarrow \infty} S_{R}(x, y)=L(x, y) \text {. }
$$

The series will be said to be circular summable $(C, \eta), \eta>0$, to the finite value $L(x, y)$ if

$$
\sigma_{R}^{(\eta)}(x, y)=\frac{2 \eta}{R^{2 \eta}} \int_{0}^{R} S_{u}(x, y)\left(R^{2}-u^{2}\right)^{\eta-1} u d u
$$

is such that $\lim _{R \rightarrow \infty} \sigma_{R}^{(\eta)}(x, y)=L(x, y)$. It has been shown by Hardy [4] that for $\eta>0$

$$
\bar{\sigma}_{R}^{(\eta)}(x, y)=\frac{\eta}{R^{\eta}} \int_{0}^{R} S_{u}(x, y)(R-u)^{\eta-1} d u
$$

and $\sigma_{R}^{(\eta)}(x, y)$ are related in the following manner: $\sigma_{R}^{(\eta)}(x, y)-L(x, y)=o(1)$ if and only if $\bar{\sigma}_{R}^{(\eta)}(x, y)-L(x, y)=o(1)$. This equivalence will be used in $\S 4$.

We shall say that the double trigonometric series $T$ is circularly summable $C$ at the point $(x, y)$ if there exists an $\eta$ such that the series is circular summable $(C, \eta)$ to a finite value at that point.

The purpose of this paper is to do for double trigonometric series that which Plessner, see Zygmund [7, pp. 256-261], has done for single series, namely to give a necessary and sufficient condition that a given double trigonometric series be circular summable $C$. This goal is achieved in Theorem 5. It will be apparent from the definitions and proofs to be given that with appropriate modifications the results of this paper could be extended to multiple trigonometric series.

Presented to the Society, February 28, 1953; received by the editors December 31, 1952.

(1) The present paper is based on Part III of the author's doctoral dissertation submitted to the Mathematics Department of the University of Chicago. The author wishes to acknowledge his indebtedness to Professor Antoni Zygmund, under whose direction the dissertation was written. 
2. Generalized Laplacians. The first step necessary in attaining the desired aim of this paper is to give a new definition for generalized Laplacians. (Though several definitions are extant in the literature, none are given in the same form as here. See, for example, Nicolesco [5] and Min Te Cheng [2].) In a manner analogous to the definition of generalized derivatives in one dimension, Zygmund [7, p. 257], we say that $f(x, y)$, defined in a neighborhood of $\left(x_{0}, y_{0}\right)$ and integrable on the circumference of every circle contained in this neighborhood with $\left(x_{0}, y_{0}\right)$ as center, has a generalized $r$ th Laplacian at $\left(x_{0}, y_{0}\right)$ equal to $\alpha_{r}$ if

$$
\begin{aligned}
\frac{1}{2 \pi} \int_{0}^{2 \pi} f\left(x_{0}+t \cos \theta, y_{0}+\right. & t \sin \theta) d \theta \\
& =\alpha_{0}+\frac{\alpha_{1} t^{2}}{[2 !]^{2}}+\frac{\alpha_{2} t^{4}}{\left[2^{2} 2 !\right]^{2}}+\cdots+\frac{\alpha_{r} t^{2 r}}{\left[2^{r} r !\right]^{2}}+o\left(t^{2 r}\right)
\end{aligned}
$$

where $t>0$ and the $\alpha_{i}$ are constants.

We shall designate the generalized $r$ th Laplacian of $f$ at $\left(x_{0}, y_{0}\right)$ by $\Delta_{r} f\left(x_{0}, y_{0}\right)$. From the definition it is clear that the existence of $\Delta_{r} f\left(x_{0}, y_{0}\right)$ implies the existence of $\Delta_{s} f\left(x_{0}, y_{0}\right)$ for $0 \leqq s \leqq r$. Designating the ordinary $r$ th Laplacian of $f$ if it exists by $\Delta^{r} f\left(x_{0}, y_{0}\right)$ where $\Delta^{r} f=\Delta\left(\Delta^{r-1} f\right), \Delta=\partial^{2} / \partial x^{2}$ $+\partial^{2} / \partial y^{2}$, and $\Delta^{0}$ is the identity operator, we have the following theorem. (See [3, p. 261] where a similar theorem is proved.)

THEOREM 1. If all partial derivatives of $f$ of order $2 r$ exist and are continuous in a neighborhood of $\left(x_{0}, y_{0}\right)$, then the generalized rth Laplacian exists and equals $\Delta^{r} f\left(x_{0}, y_{0}\right)$.

For simplicity of notation let us assume that $\left(x_{0}, y_{0}\right)$ is the origin. Then using Taylor's expansion in two variables we have for $\left(x^{2}+y^{2}\right)^{1 / 2}=t$ sufficiently small that

$$
\frac{1}{2 \pi} \int_{0}^{2 \pi} f(t \cos \theta, t \sin \theta) d \theta=f(0,0)+\sum_{j=1}^{2 r} \frac{\beta_{j}}{j !} t^{j}+o\left(t^{2 r}\right)
$$

where $\beta_{j}=\sum_{k=0}^{j}\left(\begin{array}{l}j \\ k\end{array}\right) f_{x}^{j-k} y^{k}(0,0)(1 / 2 \pi) \int_{0}^{2 \pi} \cos ^{j-k} \theta \sin ^{k} \theta d \theta$. Noticing that $\beta_{j}=0$ when $j$ is odd, we have that the generalized $r$ th Laplacian exists and consequently that

$$
\begin{aligned}
\Delta_{r} f(0,0) & =\beta_{2 r} \frac{\left[2^{r} r !\right]^{2}}{(2 r) !} \\
& =\sum_{k=0}^{r}\left(\begin{array}{l}
2 r \\
2 k
\end{array}\right) \frac{\left[2^{r} r !\right]^{2}}{(2 r) !} f_{x^{2(r-k)} y^{2 k}}(0,0) \int_{0}^{2 \pi} \cos ^{2(r-k)} \theta \sin ^{2 k} \theta d \theta \\
& =\sum_{k=0}^{r}\left(\begin{array}{l}
r \\
k
\end{array}\right) f_{x^{2(r-k)} y^{2 k}}(0,0)=\Delta^{r} f(0,0),
\end{aligned}
$$


which completes the proof.

3. The sufficiency condition for circular summability $C$. To facilitate the writing of theorems and proofs, it will be necessary at this point to introduce the following notation:

$M=(m, n), \quad X=(x, y), \quad M X=m x+n y, \quad|M|=\left(m^{2}+n^{2}\right)^{1 / 2}$.

$\Im[f]=$ the Fourier series of $f$.

$\Delta r \subseteq[f]=$ the series obtained from $\mathfrak{S}[f]$ by applying the Laplacian operator formally $r$ times.

$f_{0}(t)=(1 / 2 \pi) \int_{0}^{2 \pi} f(t \cos \theta, t \sin \theta) d \theta$.

We can now state and prove a theorem which is tantamount to the sufficiency condition for circular summability $C$.

THEOREM 2. If $\Delta_{r} f\left(X_{0}\right)$ exists, $\Delta^{r} \subseteq[f]$ is at the point $X_{0}$ circularly summable $(C, 2 \alpha), \alpha>r+1 / 4$, to the value $\Delta_{r} f\left(X_{0}\right)$.

Bochner [1] has proved this theorem for $r=0$. Throughout this proof we shall, therefore, assume that $r \geqq 1$.

Without loss of generality, we shall assume that $X_{0}$ coincides with the origin, and consequently we see that we are given

$$
f_{0}(t)=\alpha_{0}+\frac{\alpha_{1} t^{2}}{[2 !]^{2}}+\frac{\alpha_{2} t^{4}}{[222 !]^{2}}+\cdots+\frac{\alpha_{r} t^{2 r}}{[2 r r !]^{2}}+o\left(t^{2 r}\right) .
$$

We observe furthermore that there exists a trigonometric polynomial $T(X)=\xi_{0}+\xi_{1} e^{i(x+y)} \cdots+\xi_{r} e^{i r(x+y)}$ such that $\Delta^{i} T(0)=\alpha_{i}, i=0, \cdots, r$. For choose

$$
\begin{aligned}
& \xi_{0}+\xi_{1}+\quad \xi_{2}+\cdots+\quad \xi_{r}=\alpha_{0}, \\
& -\xi_{1}-\quad 2^{2} \xi_{2}-\cdots-\quad r^{2} \xi_{r}=\alpha_{1} / 2 \text {, } \\
& (-1)^{r} \xi_{1}+(-1)^{r} 2^{2 r} \xi_{2}+\cdots+(-1)^{r} r^{2 r} \xi_{r}=\alpha_{r} / 2^{r},
\end{aligned}
$$

something which clearly can be done for we have $r+1$ equations in $r+1$ unknowns, and the determinant of the coefficients is nonvanishing.

By Theorem 1, $\Delta_{i} T(0)=\alpha_{i}, \quad i=0, \cdots, r$. Furthermore $f_{0}(t)-T_{0}(t)$ $=o\left(t^{2 r}\right)$ and $\Delta^{r} \subseteq[f-T]=\Delta^{r} \subseteq[f]-\Delta^{r} \subseteq[T]=\Delta^{r} \subseteq[f]-\mathfrak{S}\left[\Delta^{r} T\right]$. Also $\mathfrak{S}\left[\Delta^{r} T\right]$ converges to $\alpha_{r}$ at the origin. Putting these last few facts together we see that there is no loss in generality in assuming that $\alpha_{0}=\alpha_{1}=\cdots=\alpha_{r}=0$. We thus have reduced the theorem to the case where $f_{0}(t)=o\left(t^{2 r}\right)$, and we therefore have to prove that $\Delta^{r} \mathfrak{S}[f]$ is circularly summable $(C, 2 \alpha)$ to zero at the origin.

Let $\subseteq[f]=\sum C_{M} e^{i M X}$. Then $\Delta^{r} \subseteq[f]=\sum(-1)^{r}|M|^{2 r} C_{M} e^{i M X}$. Designating the circular partial sum of rank $R$ of this latter series by $S_{R}(X)$, thus

$$
S_{R}(X)=\sum_{|M| \leqq R}(-1)^{r}|M|^{2 r} C_{M} e^{i M X},
$$


we see from (1) that

$$
\sigma_{R}^{(2 \alpha)}(X)=\sum_{|M| \leqq R}(-1)^{r} C_{M}|M|^{2 r}\left(1-\frac{|M|^{2}}{R^{2}}\right)^{2 \alpha} e^{i M X}=(-1)^{r} R^{2 r} T_{R}^{\phi}(X)
$$

where

$$
T_{R}^{\phi}(X)=\sum C_{M} \phi(|M| / R) e^{i M X}
$$

and

$$
\phi(u)=\left\{\begin{array}{lr}
\left(1-u^{2}\right)^{2 \alpha} u^{2 r} & \text { for } 0 \leqq u \leqq 1, \\
0 & \text { for } u>1 .
\end{array}\right.
$$

It is worth noticing at this point that $\phi(u)$ has at least two continuous derivatives in $0 \leqq u<\infty$.

For such a kernel $\phi$, we have by Bochner $[1$, Theorem V] that

$$
T_{R}^{\phi}(0)=R \int_{0}^{\infty} f_{0}(t) H_{\phi}(t R) d t
$$

where $H_{\phi}(c)=c \int_{0}^{\infty} \phi(u) u J_{0}(u c) d u$ and $J_{0}$ is the Bessel function of order zero.

We, therefore, conclude from (3) and (4) that

$$
\sigma_{R}^{(2 \alpha)}(0)=(-1)^{r} R^{2 r+1} \int_{0}^{\infty} f_{0}(t) H_{\phi}(t R) d t .
$$

Investigating $H_{\phi}$ we see that

$$
\begin{aligned}
H_{\phi}(c) & =c \int_{0}^{\pi / 2}(\cos \theta)^{4 \alpha+1}(\sin \theta)^{2 r+1} J_{0}(c \sin \theta) d \theta \\
& =c \sum_{k=0}^{r}\left(\begin{array}{l}
r \\
k
\end{array}\right)(-1)^{k} \int_{0}^{\pi / 2}(\cos \theta)^{4 \alpha+2 k+1} \sin \theta J_{0}(c \sin \theta) d \theta .
\end{aligned}
$$

By Watson [6, p. 373, formula 1] we have

$$
\int_{0}^{\pi / 2} \cos ^{4 \alpha+2 k+1} \theta \sin \theta J_{0}(c \sin \theta) d \theta=\frac{J_{2 \alpha+k+1}(c)}{c^{2 \alpha+k+1}} 2^{2 \alpha+k} \Gamma(2 \alpha+k+1) .
$$

Designating the constant

$$
(-1)^{k}\left(\begin{array}{l}
r \\
k
\end{array}\right) 2^{2 \alpha+k} \Gamma(2 \alpha+\dot{k}+1)
$$

by $b_{k}$, we have

$$
H_{\phi}(c)=\sum_{k=0}^{r} b_{k} \frac{J_{2 \alpha+k+1}(c)}{c^{2 \alpha+k}}
$$


and consequently from (5) that

$$
\sigma_{R}^{(2 \alpha)}(0)=(-1)^{r} \sum_{k=0}^{r} b_{k} R^{2 r+1} \int_{0}^{\infty} f_{0}(t) \frac{J_{2 \alpha+k+1}(t R)}{(t R)^{2 \alpha+k}} d t .
$$

We shall now show that each term of the sum on the right side of (6) is $o(1)$ as $R \rightarrow \infty$. Fix $\delta$ and let $1 / R$ be less than $\delta$. Then since $f_{0}(t)=o\left(t^{2 r}\right)$ and $J_{2 \alpha+k+1}(t R)=O\left((t R)^{2 \alpha+k+1}\right)$ as $t \rightarrow 0$ we have that

$$
\begin{array}{rlrl}
\int_{0}^{1 / R} \frac{f_{0}(t)}{t^{2 \alpha+k}} \frac{J_{2 \alpha+k+1}(t R)}{R^{2 \alpha+k}} d t & =O(R) \int_{0}^{1 / R} o\left(t^{2 r+1}\right) d t & \\
& =o\left(\frac{1}{R^{2 r+1}}\right) & & \text { as } R \rightarrow \infty
\end{array}
$$

Using the fact that there exists a constant $K$ such that

$$
\left|J_{2 \alpha+k+1}(c)\right| \leqq K / c^{1 / 2} \quad \text { for } c \geqq 1 \text { and } k=0,1, \cdots, r
$$

we have that

$$
\begin{gathered}
\int_{1 / R}^{\delta} \frac{f_{0}(t) J_{2 \alpha+k+1}(t R)}{t^{2 \alpha+k} R^{2 \alpha+k}} d t \leqq \frac{K}{R^{2 \alpha+k+1 / 2}} \int_{1 / R}^{\delta} \frac{o\left(t^{2 r}\right)}{t^{2 \alpha+k+1 / 2}} d t \\
=\frac{K}{R^{2 \alpha+k+1 / 2}} o\left(R^{2 \alpha+k-2 r-1 / 2}\right)=o\left(\frac{1}{R^{2 r+1}}\right) .
\end{gathered}
$$

Using the fact that $\int_{\delta+n}^{\delta+n+1}\left|f_{0}(t)\right| d t \leqq|A|$ where $A$ is a constant independent of $n$, we have that

$$
\begin{aligned}
\left|\int_{\delta}^{\infty} \frac{f_{0}(t) J_{2 \alpha+k+1}(t R)}{t^{2 \alpha+k} R^{2 \alpha+k}} d t\right| & \leqq \frac{K}{R^{2 \alpha+k+1 / 2}} \int_{\delta}^{\infty}\left|f_{0}(t)\right| \frac{1}{t^{2 \alpha+k+1 / 2}} d t \\
& \leqq \frac{K}{R^{2 \alpha+k+1 / 2}} \sum_{n=0}^{\infty} \int_{\delta+n}^{\delta+n+1} \frac{\left|f_{0}(t)\right|}{t^{2 \alpha+k+1 / 2}} d t \\
& =O\left(\frac{1}{R^{2 \alpha+k+1 / 2}}\right)=o\left(\frac{1}{R^{2 r+1}}\right)
\end{aligned}
$$

From (7), (8), and (9), we conclude that

$$
\int_{0}^{\infty} f_{0}(t) \frac{J_{2 \alpha+k+1}(t R)}{t^{2 \alpha+k}} d t=o\left(R^{2 r+1}\right) \quad \text { for } k=0, \cdots, r
$$

and, consequently, from (6) that $\sigma_{R}^{(2 \alpha)}(0)=o(1)$ as $R \rightarrow \infty$, which gives us the theorem.

Defining $a_{M}=o\left(|M|^{\beta}\right)$ to mean the following: given an $\epsilon>0$ there exists an $R(\epsilon)$ such that if $|M|>R$ then $\left|a_{M}\right|<\epsilon|M|^{\beta}$, we have as an immediate corollary from Theorem 2 
THEOREM 3. Let $T$ be the trigonometric series $T=\sum a_{M} e^{i M X}$ where $a_{M}$ $=o\left(|M|^{2 \gamma}\right), \gamma \geqq-1$. Apply the anti-Laplacian operator $r$ times to $T$ and $o b$ tain the series

$$
\Delta^{-r} T=\frac{a_{0}(x+y)^{2 r}}{2^{r}[(2 r) !]}+\sum_{|M| \neq 0}(-1)^{r} \frac{a_{M}}{|M|^{2 r}} e^{i M X}
$$

where $r$ is an integer greater than $\gamma+1$. Designate the sum of this series by $F(X)$. Then a sufficient condition for $T$ to be circularly summable $(C, 2 \alpha)$ at the point $X_{0}$ to the value $s, \alpha>r+1 / 4$, is that $F(X)$ have a generalized $r$ th Laplacian at that point equal to $s$.

4. The necessary condition for circular summability $C$. We shall now state and prove a theorem which will give us a necessary condition for summability $C$.

THEOREM 4. Let $T=\sum a_{M} e^{i M X}$ be a trigonometric series circularly summable $(C, 2 \alpha)$ at the point $X_{0}$ to the sum $s$ where $2 \alpha$ is an integer $\geqq 0$ and where $a_{M}=o\left(|M|^{2 \alpha-\epsilon}\right), \epsilon>0$. Let $r$ be an integer $\geqq \alpha+1$. Set

$$
F(X)=\frac{a_{0}(x+y)^{2 r}}{2^{r}[(2 r) !]}+\sum_{|M| \neq 0}(-1)^{r} \frac{a_{M}}{|M|^{2 r}} e^{i M X} .
$$

Then the generalized rth Laplacian of $F(X)$ exists at the point $X_{0}$ and is equal to $s$.

We clearly can assume from the start that $a_{0}=0$ and that $X_{0}$ is the origin. Furthermore, since there exists a trigonometric polynomial $T_{1}(X)$ without a constant term such that $T_{1}(0)=s$, we can by Theorem 1 assume that $s=0$. Also by increasing $\alpha$, if necessary, we may suppose that $r=\alpha+1$.

Using the equivalence mentioned in $\$ 1$, we see that the following two conditions are given to us:

$$
\begin{aligned}
& \tilde{\sigma}_{R}^{(2 \alpha)}(0)=\sum_{|M| \leqq R} a_{M}\left(1-\frac{|M|}{R}\right)^{2 \alpha}=o(1) \quad \text { as } R \rightarrow \infty, \\
& (-1)^{r} \sum_{|M| \leqq R} \frac{a_{M}}{|M|^{2 r}} e^{i M X} \rightarrow F(X) \text { uniformly in } x \text { and } y,
\end{aligned}
$$

where $a_{0}=0$ and $\tilde{\sigma}_{R}^{(0)}(0)$ is to be interpreted as $S_{R}(0)$ in case $\alpha=0$. Clearly,

$$
\frac{1}{2 \pi} \int_{0}^{2 \pi} e^{i t(m \cos \theta+n \sin \theta)} d \theta=J_{0}(|M| t) .
$$

From this fact and (11), we conclude that

$$
F_{0}(t)=(-1) r \sum_{M} a_{M} \frac{J_{0}(|M| t)}{|M|^{2 r}}
$$


Setting $\gamma(u)=J_{0}(u) / u^{2 r}$ for $u>0$, we see that (12) can be written as

$$
F_{0}(t)=(-1)^{r} t^{2 r} \sum_{M} \gamma(|M| t) a_{M} \quad \text { for } t>0 .
$$

It is necessary at this point to prove some lemmas. We shall adopt the following conventions in so doing:

$1^{\circ} . a_{0}=0$.

$2^{\circ}$. $S_{R}=S_{R}^{(0)}=S_{R}(0)=\sum_{|M| \leqq R} a_{M}$.

$3^{\circ}$. $S_{R}^{(k)}=\int_{1}^{R} S_{u}^{(k-1)} d u$.

$4^{\circ}$. $\gamma^{(k)}(u)=d^{k} \gamma(u) / d u^{k} ; \gamma^{(0)}(u)=\gamma(u)$.

It is to be noticed also that

$$
\bar{\sigma}_{R}^{(k)}=k ! S_{R}^{(k)} / R^{k} \text {. }
$$

LEMMA 1. $\sum_{|M| \leqq R} a_{M} \gamma(|M| t)=-\int_{1}^{R} S_{u}(d \gamma(u t) / d u) d u+S_{R} \gamma(R t)$ for $t>0$.

To prove the lemma define an additive function of sets $\chi$, additive $(B)$ on every figure in the plane in the following manner:

$\chi(M)=a_{m n}$, where $M$ is a lattice point.

$\chi\left(\mathrm{U}_{M} M\right)=\mathrm{U}_{\chi}(M)$ for any finite union of lattice points.

$\chi(E)=\chi(E \cap \Lambda)$, where $E$ is any bounded Borel set and $\Lambda$ is the set of lattice points in the plane.

$\chi$ is clearly an additive function of sets defined on the Borel sets of any figure, and if we designate by $C_{R}$ the closed circle of radius $R$ with center at the origin, we have that $S_{R}=\int_{C_{R}} d \chi(P)$.

Now letting $f_{C_{u}}(P)$ stand for the characteristic function of $C_{u}$ and $|P|$ $=\left(p^{2}+q^{2}\right)^{1 / 2}$, we have that

$$
\begin{aligned}
\int_{1}^{R} S_{u} \frac{d \gamma(u t)}{d u} d u & =\int_{1}^{R} \frac{d \gamma(u t)}{d u} d u \int_{C_{u}} d \chi(P) \\
& =\int_{1}^{R} \frac{d \gamma(u t)}{d u} d u \int_{C_{R}} f_{C_{u}}(P) d \chi(P) \\
& =\int_{C_{R}} d \chi(P) \int_{1}^{R} f_{C_{u}}(P) \frac{d \gamma(u t)}{d u} d u \\
& =\int_{C_{R}} d \chi(P) \int_{|P|}^{R} \frac{d \gamma(u t)}{d u} d u \\
& =\int_{C_{R}}[\gamma(R t)-\gamma(|P| t)] d \chi(P) \\
& =-\sum_{|M| \leqq R} a_{M} \gamma(|M| t)+\gamma(R t) S_{R},
\end{aligned}
$$

which proves this lemma. 
LEMMA 2. $\gamma^{(k)}(u)=o\left(u^{-2 r}\right)$ as $u \rightarrow \infty$ for $k=0,1, \cdots, 2 \alpha+1$.

Write

$$
\frac{d^{k} \gamma(u)}{d u^{k}}=\frac{d^{k}\left(J_{0}(u) / u^{2 r}\right)}{d u^{k}}=\sum_{j=0}^{k} \frac{b_{j}}{u^{2 r+k-j}} \frac{d^{j} J_{0}(u)}{d u^{j}}
$$

where $b_{j}$ are constants, $j=0, \cdots, k$. Now

$$
\frac{d^{j} J_{0}(u)}{d u^{j}}=\frac{1}{2^{j}} \sum_{m=0}^{j}(-1)^{m}\left(\begin{array}{l}
j \\
m
\end{array}\right) J_{2 m-j}(u)
$$

by Watson [6, p. 18, formula 8]. Since $J_{2 m-j}(u)=o(1)$, we have that $d^{i} J_{0}(u) / d u^{j}=o(1)$ and consequently from (15) that $d^{k} \gamma(u) / d u^{k}=o\left(1 / u^{2 r}\right)$.

Lemma 3. There exists a set of constants $C_{j k}, j=1, \cdots, 2 \alpha+1, k=0, \cdots$ ' $2 \alpha$, such that $\sum_{j=1}^{2 \alpha+1} C_{j k}(z+j)^{2 \alpha}=z^{k}$ for all complex numbers $z$.

The proof of this lemma follows readily from a consideration of Vandermonde determinants.

LemmA 4. Let $a_{M}=o\left(|M|^{2 \alpha-\epsilon}\right), \epsilon>0$. Suppose $S_{R}$ is summable $(C, 2 \alpha)$ to zero. Then $S_{R}^{(k)}=o\left(R^{2 \alpha+1}\right)$ for $k=0,1, \cdots, 2 \alpha$.

Since there is nothing to prove if $2 \alpha=0$, we shall assume that $2 \alpha$ is an integer greater than zero.

By the equivalence between $\bar{\sigma}_{R}^{(2 \alpha)}$ and $\sigma_{R}^{(2 \alpha)}$ mentioned in the introduction and by (10), we see that

$$
\sum_{|M| \leqq R} a_{M}(R-|M|)^{2 \alpha}=o\left(R^{2 \alpha}\right) .
$$

Observing that the number of lattice points in the annulus determined by the circles $C_{R+2 \alpha+1}$ and $C_{R}$ is $O(R)$ as $R \rightarrow \infty$, where $C_{K}$ is a circle with center at the origin and radius $K$, we also see that for $j=1,2, \cdots, 2 \alpha+1$

$$
\begin{aligned}
\sum_{|M| \leqq R} a_{M}(R-|M|+i)^{2 \alpha}= & \sum_{|M| \leqq R+j} a_{M}(R+j-|M|)^{2 \alpha} \\
& -\sum_{R<|M| \leqq R+j} a_{M}(R+j-|M|)^{2 \alpha} \\
& =o\left(R^{2 \alpha}\right)-o\left(R^{2 \alpha}\right) O(R)=o\left(R^{2 \alpha+1}\right) .
\end{aligned}
$$

Now by (10), (14), and Lemma 3

$$
\begin{array}{r}
S_{R}^{(k)}=\frac{1}{k !} \sum_{|M| \leqq R} a_{M}(R-|M|)^{k}=\frac{1}{k !} \sum_{|M| \leqq R} a_{M} \sum_{j=1}^{2 \alpha+1} C_{j k}(R-|M|+j)^{2 \alpha} \\
\quad \text { for } k=0, \cdots, 2 \alpha .
\end{array}
$$


Therefore

$$
S_{R}^{(k)}=\frac{1}{k !} \sum_{j=1}^{2 \alpha+1} C_{j k} \sum_{|M| \leqq R} a_{M}(R-|M|+j)^{2 \alpha}=o\left(R^{2 \alpha+1}\right)
$$

by (16), which gives us the lemma.

Returning now to the proof of the theorem, we obtain from (13) and Lemma 1 that

$$
F_{0}(t)=(-1)^{r+1} t^{2 r} \lim _{R \rightarrow \infty}\left(\int_{1}^{R} S_{u} \frac{d \gamma(u t)}{d u} d u-S_{R} \gamma(R t)\right) .
$$

Integrating the integral on the right of (17) by parts $2 \alpha$ times, we have

$$
\begin{aligned}
\int_{1}^{R} S_{u} \frac{d \gamma(u t)}{d u} d u= & \sum_{k=1}^{2 \alpha}(-1)^{k+1} S_{R}^{(k)} t^{k} \gamma^{(k)}(R t) \\
& +\int_{1}^{R} S_{u}^{(2 \alpha)} \frac{d^{2 \alpha+1}}{d u^{2 \alpha+1}} \gamma(u t \iota .
\end{aligned}
$$

By Lemma 2, $\gamma^{(k)}(R t)=o\left(R^{-2 r}\right)$ for fixed $t$ and $k=0, \cdots, 2 \alpha$. By Lemma $4, S_{R}^{(k)}=o\left(R^{2 \alpha+1}\right)=o\left(R^{2 r}\right)$ for the same $k$. Therefore from (17) and (18) we conclude that

$$
F_{0}(t)=(-1)^{r+1} t^{2 r} \int_{1}^{\infty} S_{u}^{(2 \alpha)} \frac{d^{2 \alpha+1}}{d u^{2 \alpha+1}} \gamma(u t) d u .
$$

As is well known,

$$
J_{0}(u)=1-u^{2} / 2^{2}+\cdots+(-1)^{n} u^{2 n} / 2^{2} 4^{2} \cdots(2 n)^{2}+\cdots .
$$

Define

$$
P(u)=1-\frac{u^{2}}{2^{2}}+\cdots+\frac{(-1)^{r-1} u^{2(r-1)}}{2^{2} 4^{2} \cdots[2(r-1)]^{2}}=\sum_{i=0}^{r-1} b_{i} u^{2 i}
$$

where $b_{i}=(-1)^{i} / 2^{2} \cdots(2 i)^{2}, \quad i=1, \cdots, r-1$, and $b_{0}=1$. Set $\lambda(u)$ $=\left(J_{0}(u)-P(u)\right) / u^{2 r}$. Then $\lambda(u)$ is a function which is regular in the plane. Furthermore $\lambda(u t)=\gamma(u t)-P(u t) /(u t)^{2 r}$. Therefore

$$
\frac{d^{2 \alpha+1} \gamma(u t)}{d u^{2 \alpha+1}}=\frac{d^{2 \alpha+1} \lambda(u t)}{d u^{2 \alpha+1}}+\frac{d^{2 \alpha+1}\left(P(u t) /(u t)^{2 r}\right)}{d u^{2 \alpha+1}} .
$$

But

$$
\frac{d^{2 \alpha+1}\left(P(u t) /(u t)^{2 r}\right)}{d u^{2 \alpha+1}}=\sum_{i=0}^{r-1} b_{i} t^{2(i-r)} u^{2(i-r-\alpha)-1}
$$

where $b_{i}^{\prime}$ are constants, $i=0, \cdots, r-1$. It is also to be noticed that by hypothesis $S_{u}^{(2 \alpha)}=(1 /(2 \alpha) !) \sum_{|M| \leqq u} a_{M}(u-|M|)^{2 \alpha}=o\left(u^{2 \alpha}\right)$. We see, therefore, from (19), (20), and (21) that 


$$
\begin{aligned}
F_{0}(t)= & (-1)^{r+1} t^{2 r} \int_{1}^{\infty} S_{u}^{(2 \alpha)}\left[\frac{d^{2 \alpha+1}\left(P(u t) /(u t)^{2 r}\right)}{d u^{2 \alpha+1}}+\frac{d^{2 \alpha+1} \lambda(u t)}{d u^{2 \alpha+1}}\right] d u \\
= & (-1)^{r+1} \sum_{i=0}^{r-1} t^{2 i} b_{i}^{\prime} \int_{1}^{\infty} S_{u}^{(2 \alpha)} u^{2(i-r-\alpha)-1} d u \\
& +(-1)^{r+1} t^{2 r} \int_{1}^{\infty} S_{u}^{(2 \alpha)} \frac{d^{2 \alpha+1} \lambda(u t)}{d u^{2 \alpha+1}} d u
\end{aligned}
$$

each of the integrals in the sum on the right being clearly absolutely convergent.

$F_{0}(t)$ is, therefore, expressible in the form of

$$
\begin{aligned}
F_{0}(t)=\alpha_{0} & +\frac{\alpha_{1} t^{2}}{[2 !]^{2}}+\cdots+\frac{\alpha_{r-1} t^{2(r-1)}}{\left[2^{r-1}(r-1) !\right]^{2}} \\
& +(-1)^{r+1}\left[\int_{1}^{\infty} S_{u}^{(2 \alpha)} \frac{d^{2 \alpha+1} \lambda(u t)}{d u^{2 \alpha+1}} d u\right] t^{2 r}
\end{aligned}
$$

and the theorem will be proved when it is shown that the integral on the right of (23) is $o(1)$ as $t \rightarrow 0$. We split this integral into two parts, obtaining

$$
A=\int_{1}^{1 / t} S_{u}^{(2 \alpha)} \frac{d^{2 \alpha+1} \lambda(u t)}{d u^{2 \alpha+1}} d u, \quad B=\int_{1 / t}^{\infty} S_{u}^{(2 \alpha)} \frac{d^{2 \alpha+1} \lambda(u t)}{d u^{2 \alpha+1}} d u .
$$

We shall show that both $A$ and $B$ are $o(1)$.

Since $\lambda(z)$ is a function regular in the plane, there exists a constant $K$ such that $\left|\lambda^{(2 \alpha+1)}(z)\right| \leqq K$ for $|z| \leqq 1$. Consequently

$$
\left|\frac{d^{2 \alpha+1}}{d u^{2 \alpha+1}} \lambda(u t)\right|=\left|t^{2 \alpha+1} \lambda^{(2 \alpha+1)}(u t)\right| \leqq K t^{2 \alpha+1}
$$

for $|u t| \leqq 1$ and $t>0$. Therefore

$$
|A| \leqq K t^{2 \alpha+1} \int_{1}^{1 / t} o\left(u^{2 \alpha}\right) d u=o(1)
$$

To show that $B$ is $o(1)$, we observe that

$$
\frac{d^{2 \alpha+1}}{d u^{2 \alpha+1}} \lambda(u t)=\frac{d^{2 \alpha+1}}{d u^{2 \alpha+1}} \gamma(u t)-\frac{d^{2 \alpha+1}\left(P(u t) /(u t)^{2 r}\right)}{d u^{2 \alpha+1}}
$$

and by Lemma 2 that for $|u t| \geqq 1$

$$
\left|\frac{d^{2 \alpha+1}}{d u^{2 \alpha+1}} \gamma(u t)\right|=t^{2 \alpha+1}\left|\gamma^{(2 \alpha+1)}(u t)\right| \leqq \frac{t^{2 \alpha+1} K}{u^{2 r} t^{2 r}}=\frac{1}{t} \frac{K}{u^{2 r}},
$$

where $K$ is a constant. 
We furthermore observe that

$$
\frac{1}{t} \int_{1 / t}^{\infty} o\left(u^{2 \alpha}\right) \frac{d u}{u^{2 r}}=\frac{1}{t} \int_{1 / t}^{\infty} o\left(\frac{1}{u^{2}}\right) d u=o(1) \quad \text { as } t \rightarrow 0
$$

and that

$$
t^{2(i-r)} \int_{1 / t}^{\infty} o\left(u^{2 \alpha}\right) u^{2(i-r-\alpha)-1} d u=o(1) \quad \text { for } i=0, \cdots, r-1
$$

as $t \rightarrow 0$ and consequently that

$$
B=\int_{1 / t}^{\infty} S_{u}^{(2 \alpha)} \frac{d^{2 \alpha+1}}{d u^{2 \alpha+1}} \lambda(u t) d u=o(1)
$$

by (21), (24), and (25), which fact concludes the proof of the theorem.

5. Statement of main result. From Theorems 3 and 4 , we obtain the following theorem which constitutes the desired goal of this paper.

THEOREM 5. Let $T=\sum a_{m n} e^{i(m x+n y)}$ be a double trigonometric series with coefficients $a_{m n}=o\left(\left(m^{2}+n^{2}\right)^{\gamma}\right), \gamma \geqq-1$. A necessary and sufficient condition that the series should be circularly summable $C$ at the point $\left(x_{0}, y_{0}\right)$ to the sum $s$ is that there exists an integer $r>\gamma+1$ such that if

$$
F(x, y)=\frac{a_{00}(x+y)^{2 r}}{2^{r}[(2 r) !]}+\sum_{|M| \neq 0} \frac{(-1)^{r} a_{m n}}{\left(m^{2}+n^{2}\right)^{r}} e^{i(m x+n y)}
$$

then $\Delta_{r} F\left(x_{0}, y_{0}\right)$ exists and is equal to $s$.

\section{BIBLIOGRAPHY}

1. S. Bochner, Summation of multiple Fourier series by spherical means, Trans. Amer. Math. Soc. vol. 40 (1936) pp. 175-207.

2. M. T. Cheng, On a theorem of Nicolesco and generalized Laplace operators, Proc. Amer. Math. Soc. vol. 2 (1951) pp. 77-85.

3. R. Courant and D. Hilbert, Methoden der mathematischen Physik II, Berlin, 1937.

4. G. H. Hardy, The second theorem of consistency for summable series, Proc. London Math. Soc. (2) vol. 15 (1916) pp. 72-88.

5. M. Nicolesco, Les fonctions polyharmoniques, Exposés sur la théorie des fonctions, vol. 4 , 1936, Paris.

6. G. N. Watson, Theory of Bessel functions, Cambridge, 1944.

7. A. Zygmund, Trigonometrical series, Warsaw, 1935.

University of Chicago,

Chicago, Ill.

RUTGERS UNIVERSITY,

New Brunswick, N. J. 